\title{
Kleinberg sequences and partition cardinals below $\delta_{5}^{1}$
}

\author{
by
}

Benedikt Löwe (Bonn)

\begin{abstract}
The author computes the Kleinberg sequences derived from the three different normal ultrafilters on $\boldsymbol{\delta}_{3}^{1}$.
\end{abstract}

1. Introduction. Eugene Kleinberg linked the theory of partition cardinals to the Axiom of Determinacy AD by showing that the first $\omega+1$ infinite cardinals satisfy certain large cardinal properties defined via partition relations. In fact, his proof did not actually use the Axiom of Determinacy but some of its consequences.

More generally, Kleinberg showed (for a proof, cf. [Kl77], or [Sch99] for a more thorough presentation):

THEOREM 1.1. Let $\kappa$ be a cardinal with the strong partition property and $\mu$ be a normal ultrafilter on $\kappa$. Let $\kappa_{1}:=\kappa$ and $\kappa_{n+1}:=\left(\kappa_{n}\right)^{\kappa} / \mu$. Then

(i) $\kappa_{1}$ and $\kappa_{2}$ are measurable,

(ii) for all $n \geq 2, \operatorname{cf}\left(\kappa_{n}\right)=\kappa_{2}$,

(iii) $\kappa_{n}$ is a Jónsson cardinal, and

(iv) $\sup \left\{\kappa_{n}: n \in \omega\right\}$ is a Rowbottom cardinal.

Moreover, if $\kappa^{\kappa} / \mu=\kappa^{+}$, then $\kappa_{n+1}=\left(\kappa_{n}\right)^{+}$for all $n \in \omega$.

Corollary 1.2. Assume AD. Then for all positive natural numbers $n$, $\aleph_{n}$ is a Jónsson cardinal and $\aleph_{\omega}$ is a Rowbottom cardinal.

Proof. After a brief look at Theorem 1.1 we realize that there is nothing to show if $\aleph_{1}$ has the strong partition property and $\left(\aleph_{1}\right)^{\aleph_{1}} / \mu=\aleph_{2}$ for some (the only) normal ultrafilter $\mu$ on $\aleph_{1}$. But the first assertion is a theorem of

2000 Mathematics Subject Classification: Primary 03E55, 03E60; Secondary 03C20.

The author would like to thank Ute Schmid (Tübingen) whose [Sch99, Kapitel 5, (3)] was the source of the question solved in this note, and Steve Jackson (Denton, TX) who provided the information about the cofinalities below $\boldsymbol{\delta}_{5}^{1}$. Instrumental for the results in this paper was the author's attendance at the 1998-99 ASL Annual Meeting in San Diego, CA and the AMS Western Sectional Meeting in Las Vegas, NV, which were funded by an ASL Travel Grant and a Sonderbeihilfe of the Studienstiftung des deutschen Volkes. 
Martin (cf. [Ka94, Theorem 28.12]), the second is a theorem of Solovay (cf. [Kl77, Theorem 2.9]).

At that time, it was unknown whether there are any natural assumptions (e.g., the Axiom of Determinacy) under which the conditions of Kleinberg's Theorem 1.1 are met except for the case mentioned in the proof of Corollary 1.2 .

The deep structural results of Jackson's computation of $\boldsymbol{\delta}_{5}^{1}$ immediately provided additional examples for Kleinberg's theorem under AD: All odd projective ordinals $\boldsymbol{\delta}_{2 n+1}^{1}$ are starting points for sequences of successive Jónsson cardinals derived from the $\omega$-cofinal normal ultrafilter (cf. Theorem 2.6 and Fact 2.5(vii)).

But Kleinberg's Theorem 1.1 provides us with even more sequences of Jónsson cardinals starting from $\boldsymbol{\delta}_{2 n+1}^{1}$ since we have as many normal measures on $\boldsymbol{\delta}_{2 n+1}^{1}$ as we have regular cardinals below it. Where exactly are these Jónsson and Rowbottom cardinals? Can we compute the cardinality of the members of these additional Kleinberg sequences?

In this note we shall answer these questions and compute the Kleinberg sequences derived from the $\omega_{1}$-cofinal and the $\omega_{2}$-cofinal measures on $\boldsymbol{\delta}_{3}^{1}$. An important ingredient here is the exact knowledge of cofinalities of successor cardinals between $\boldsymbol{\delta}_{3}^{1}$ and $\boldsymbol{\delta}_{5}^{1}$ provided by [JaKh $\infty$ ].

2. Prerequisites and the Shifting Lemma. To compute the Kleinberg sequences, we will use a substantial amount of knowledge about the behaviour of the projective ordinals and of the combinatorial theory below $\boldsymbol{\delta}_{5}^{1}$ under AD. Nevertheless, we try to keep the paper understandable for readers with a basic understanding of Determinacy and Large Cardinals by listing all theorems that we shall use later on in this section.

Definition 2.1. A cardinal $\kappa$ is called a Jónsson cardinal if the partition relation $\kappa \rightarrow[\kappa]_{\kappa}^{<\omega}$ holds, i.e., for every partition of $[\kappa]^{<\omega}$ into $\kappa$ blocks there is a set $H$ of order type $\kappa$ with the property that $[H]^{<\omega}$ does not meet all blocks.

A cardinal $\kappa$ is called a Rowbottom cardinal if for all $\lambda<\kappa$ the partition relation $\kappa \rightarrow[\kappa]_{\lambda,<\omega}^{<\omega}$ holds, i.e., for every partition of $[\kappa]^{<\omega}$ into $\lambda$ blocks there is a set $H$ of order type $\kappa$ with the property that $[H]^{<\omega}$ meets only countably many blocks.

Jónsson and Rowbottom cardinals are large cardinals in the sense that their existence implies the consistency of ZFC (and much more). They are not, however, large in the usual sense. They do not even have to be regular cardinals; in fact, all of the Jónssons and Rowbottoms appearing in this paper have cofinality $\omega$. 
This is not just a feature of choiceless set theory: In the Př́krý (ZFC)model obtained by generically adding a cofinal $\omega$-sequence to a measurable cardinal, the former measurable cardinal is a Rowbottom cardinal of cofinality $\omega$. For particular instances of the question "Is $\aleph_{\lambda}$ Rowbottom?" where $\lambda$ is of cofinality $\omega$, the consistency strength of a positive answer differs depending on whether or not you demand that the Axiom of Choice AC holds (cf. [Koe88] and [ApKoe $\infty]$ ).

A reader interested in the basic theory of Jónsson and Rowbottom cardinals is referred to [Ka94, $\S 7 \& \S 8]$.

Definition 2.2. A cardinal $\kappa$ is said to have the strong partition property if the partition relation $\kappa \rightarrow(\kappa)^{\kappa}$ holds, i.e., if for every partition of $[\kappa]^{\kappa}$ into two blocks there is a homogeneous set of order type $\kappa$.

Note that the strong partition property cannot hold for any cardinal if we assume AC: by a result of Erdős and Rado (cf. [Ka94, Proposition 7.1]) no partition relation can have infinite exponents if the Axiom of Choice holds.

That the strong partition property of $\kappa$ really is a property with astonishing consequences for the combinatorial theory of $\kappa$ (or, to put it in Jim Henle's words, that it is "one of the most powerful partition properties known to man" [He79, p. 151]), can be seen in the next result of Kleinberg; a proof can be found in [Ka94, Theorem 28.10 \& Exercise 28.11]:

THEOREM 2.3. Let $\kappa$ be a cardinal with the strong partition property and $\lambda<\kappa$ a regular cardinal. Then $\mathcal{C}_{\kappa}^{\lambda}$, the filter generated by the $\lambda$-closed unbounded sets in $\kappa$, is a normal ultrafilter on $\kappa$. We call $\mathcal{C}_{\kappa}^{\lambda}$ the $\lambda$-cofinal filter or measure.

In addition, if $\kappa$ is not weakly Mahlo, then these are the only normal ultrafilters on $\kappa$.

The reader was already informally introduced to Kleinberg sequences in Theorem 1.1. Now we fix our notation:

DEFINITION 2.4. Let $\kappa$ be a cardinal with the strong partition property and $\mu$ a normal measure on $\kappa$. We then define a sequence of well-ordered structures $\left\langle\kappa_{n}^{\mu}: n \leq \omega\right\rangle$ as follows:

- $\kappa_{1}^{\mu}:=\kappa$,

- $\kappa_{n+1}^{\mu}:=\left(\kappa_{n}^{\mu}\right)^{\kappa} / \mu$, and

- $\kappa_{\omega}^{\mu}:=\sup \left\{\kappa_{n}^{\mu}: n \in \omega\right\}$.

This sequence is called the Kleinberg sequence derived from $\mu$.

As already mentioned in Theorem 1.1, all elements of a Kleinberg sequence are Jónsson cardinals, and $\kappa_{\omega}$ is a Rowbottom cardinal.

We define the projective ordinals by

$\boldsymbol{\delta}_{n}^{1}:=\sup \left\{\xi: \xi\right.$ is the length of a prewellordering of $\omega^{\omega}$ in $\left.\boldsymbol{\Delta}_{n}^{1}\right\}$. 
Even before Jackson's results, a couple of things were known about the projective ordinals under AD:

FACT 2.5. Let $n$ be a natural number. Assume AD. Then:

(i) (Kunen-Martin 1971) $\delta_{2 n+2}^{1}=\left(\delta_{2 n+1}^{1}\right)^{+}$,

(ii) (Kechris 1974) $\delta_{2 n+1}^{1}$ is the cardinal successor of a cardinal of cofinality $\omega$,

(iii) (Martin-Kunen 1971) all $\boldsymbol{\delta}_{n}^{1}$ are measurable,

(iv) (Martin-Kunen 1971) $\boldsymbol{\delta}_{2}^{1}=\aleph_{2}, \boldsymbol{\delta}_{3}^{1}=\aleph_{\omega+1}$, and $\boldsymbol{\delta}_{4}^{1}=\aleph_{\omega+2}$,

(v) (Martin, Paris 1971) $\delta_{1}^{1} \rightarrow\left(\delta_{1}^{1}\right)^{\delta_{1}^{1}}$, and for all $\alpha<\delta_{2}^{1}$, the relation $\delta_{2}^{1} \rightarrow\left(\delta_{2}^{1}\right)^{\alpha}$ holds

(vi) (Martin 1971) for all $\alpha<\omega_{1}$ the partition relation $\boldsymbol{\delta}_{2 n+1}^{1} \rightarrow\left(\delta_{2 n+1}^{1}\right)^{\alpha}$ holds,

(vii) (Kunen 1971) the $\omega$-cofinal measure $\mathcal{C}_{\delta_{2 n+1}^{1}}^{\omega}$ is a normal measure on $\boldsymbol{\delta}_{2 n+1}^{1}$ with $\left(\boldsymbol{\delta}_{2 n+1}^{1}\right)^{\delta_{2 n+1}^{1}} / \mathcal{C}_{\delta_{2 n+1}^{1}}^{\omega}=\delta_{2 n+2}^{1}$, and

(viii) (Martin-Jackson 1980) $\left(\delta_{3}^{1}\right)^{\delta_{3}^{1}} / \mathcal{C}_{\delta_{3}^{1}}^{\omega_{1}}=\aleph_{\omega \cdot 2+1}$ and $\left(\delta_{3}^{1}\right)^{\delta_{3}^{1}} / \mathcal{C}_{\delta_{3}^{1}}^{\omega_{2}}=$ $\aleph_{\omega^{\omega}+1}$, and these two cardinals are measurable.

Proof. A proof of all parts except for the last can be found in [Ke78]. Fact 2.5 comprises Theorems $3.12,3.10,5.1, \S 6$, Theorem 12.1, Corollary 13.4, Theorems 11.2, 14.3 of [Ke78]. The last assertion is part of [Ja99b, Chapter 7].

Since the values of $\boldsymbol{\delta}_{1}^{1}, \boldsymbol{\delta}_{2}^{1}, \boldsymbol{\delta}_{3}^{1}$, and $\boldsymbol{\delta}_{4}^{1}$ were known, the next open question was the value of $\boldsymbol{\delta}_{5}^{1}$. This was the content of the First Victoria Delfino Problem (cf. [KeMo78]), and was solved by Steve Jackson who computed $\boldsymbol{\delta}_{5}^{1}$ to be $\aleph_{\omega^{\omega} \omega}{ }^{\omega}$ (cf. [Ja88] and [Ja99b]):

TheOREM 2.6. Assume AD. Let $E$ be the function recursively defined by $E(0)=1$ and $E(n+1)=\omega^{E(n)}$. Then for every $n \in \omega$,

$$
\delta_{2 n+1}^{1}=\aleph_{E(2 n+1)+1},
$$

and all odd projective ordinals have the strong partition property.

This computation gave rise to a detailed analysis of the cardinals between $\delta_{3}^{1}$ and $\delta_{5}^{1}$ that will be used in this note.

The main tool of our computation will be the following theorem, which is an elaboration of the proof of the "moreover" part in Theorem 1.1:

Ultrapower Shifting Lemma 2.7. Let $\kappa=\aleph_{\alpha}<\lambda=\aleph_{\alpha+\beta}$, and let $\mu$ be a $\kappa$-complete ultrafilter on $\kappa$. Let $\gamma$ be such that $\kappa^{\kappa} / \mu=\aleph_{\gamma}$. Suppose that for all cardinals $\nu$ such that $\kappa<\nu \leq \lambda$ the following holds:

(i) either $\nu$ is a successor and $\operatorname{cf}(\nu)>\kappa$,

(ii) or $\nu$ is a limit and $\operatorname{cf}(\nu)<\kappa$.

Then $\lambda^{\kappa} / \mu \leq \aleph_{\gamma+\beta}$. 
Proof. The proof proceeds by induction on $\beta$. The case $\beta=0$ is just the definition of $\gamma$.

For the successor step suppose that $\lambda=\aleph_{\alpha+\beta+1}$ and that $\left(\aleph_{\alpha+\beta}\right)^{\kappa} / \mu \leq$ $\aleph_{\gamma+\beta}$. Pick any $\eta \in \lambda^{\kappa} / \mu$. Let $f: \kappa \rightarrow \lambda$ be a function representing $\eta$, so $\eta=[f]_{\mu}$. Since $\operatorname{cf}(\lambda)>\kappa$, we know that $\operatorname{ran}(f)$ is bounded in $\lambda$, say by $\eta^{*}<\lambda$. Hence $\eta \in\left(\eta^{*}\right)^{\kappa} / \mu$.

$\operatorname{But} \operatorname{Card}\left(\left(\eta^{*}\right)^{\kappa} / \mu\right)=\operatorname{Card}\left(\operatorname{Card}\left(\eta^{*}\right)^{\kappa} / \mu\right) \leq \aleph_{\gamma+\beta}$ by the induction hypothesis. Thus every ordinal in $\lambda^{\kappa} / \mu$ has cardinality $\leq \aleph_{\gamma+\beta}$, and consequently, $\lambda^{\kappa} / \mu \leq \aleph_{\gamma+\beta+1}$.

Now we look at the limit step, where $\chi$ is a limit ordinal and for all $\beta<\chi$ we have $\left(\aleph_{\alpha+\beta}\right)^{\kappa} / \mu \leq \aleph_{\gamma+\beta}$. We show that $\left(\aleph_{\alpha+\chi}\right)^{\kappa} / \mu=\bigcup_{\beta<\chi}\left(\aleph_{\alpha+\beta}\right)^{\kappa} / \mu$. This shows the claim, since

$$
\begin{aligned}
\operatorname{Card}\left(\bigcup_{\beta<\chi}\left(\aleph_{\alpha+\beta}\right)^{\kappa} / \mu\right) & \leq \sup \left\{\operatorname{Card}\left(\left(\aleph_{\alpha+\beta}\right)^{\kappa} / \mu\right): \beta<\chi\right\} \\
& \leq \sup \left\{\aleph_{\gamma+\beta}: \beta<\chi\right\}=\aleph_{\gamma+\chi} .
\end{aligned}
$$

As the backward inclusion is clear, we proceed to the other direction. Take $\eta \in\left(\aleph_{\alpha+\chi}\right)^{\kappa} / \mu$ and a function $f: \kappa \rightarrow \aleph_{\alpha+\chi}$ with $[f]_{\mu}=\eta$. Let $\left\langle B_{\delta}\right.$ : $\delta<\operatorname{cf}(\chi)\rangle$ be a partition of $\aleph_{\alpha+\chi}$ into sets of cardinality $\operatorname{Card}\left(B_{\delta}\right)<\aleph_{\alpha+\chi}$ none of which is cofinal in $\aleph_{\alpha+\chi}$ (e.g., the intervals determined by a cofinal sequence of length $\operatorname{cf}(\chi))$.

Now define $F_{\delta}:=\left(f^{-1}\right) " B_{\delta}$. Then $\left\langle F_{\delta}: \delta<\operatorname{cf}(\chi)\right\rangle$ is a disjoint partition of $\kappa$ into less than $\kappa$ sets (by assumption on $\operatorname{cf}(\chi)$ ), hence by $\kappa$-completeness there is a $\delta_{0}$ such that $F_{\delta_{0}} \in \mu$.

But $B_{\delta_{0}}$ was not cofinal in $\aleph_{\alpha+\chi}$, so we can set $\beta_{0}:=\sup \left(B_{\delta_{0}}\right)+1<\aleph_{\alpha+\chi}$, and define $f_{0}(\xi):=\min \left(f(\xi), \beta_{0}\right)$. Let $\beta_{1}<\chi$ be the unique ordinal such that $\operatorname{Card}\left(\beta_{0}\right)=\aleph_{\alpha+\beta_{1}}$. Then $f_{0}: \kappa \rightarrow \aleph_{\alpha+\beta_{1}+1}$ and $\left[f_{0}\right]_{\mu}=[f]_{\mu}$, hence $\eta \in\left(\aleph_{\alpha+\beta_{1}+1}\right)^{\kappa} / \mu$.

Note that the assumption of $\kappa$-completeness is only used in the limit step. Consequently, if we strengthen assumption (ii) to " $\nu$ is a limit and $\operatorname{cf}(\nu)<\eta$ " for some $\eta<\kappa$, we can weaken the completeness assumption to $\eta$-completeness. This is particularly interesting in the case $\eta=\omega_{1}$, because $\omega_{1}$-completeness of any measure is a consequence of "All sets of reals are Lebesgue measurable" (and thus of AD). So, in the base theory ZF + AD, we do not have to make any completeness assumptions if the limit cardinals occurring in the applications of the Ultrapower Shifting Lemma have cofinality $\omega$.

3. Computations of the Kleinberg sequences. By Theorem 2.3, we have exactly three normal ultrafilters $\mu_{0}:=\mathcal{C}_{\delta_{3}^{1}}^{\omega}, \mu_{1}:=\mathcal{C}_{\delta_{3}^{1}}^{\omega_{1}}$, and $\mu_{2}:=\mathcal{C}_{\delta_{3}^{1}}^{\omega_{2}}$ on $\boldsymbol{\delta}_{3}^{1}$, corresponding to the three regular cardinals $\aleph_{0}, \aleph_{1}$, and $\aleph_{2}$ below $\boldsymbol{\delta}_{3}^{1}$. 
Using the fact that $\boldsymbol{\delta}_{3}^{1}$ has the strong partition property by Theorem 2.6 and Kleinberg's Theorem 1.1, we obtain three Kleinberg sequences $\left\langle\kappa_{n}^{\mu_{0}}: n \leq \omega\right\rangle$, $\left\langle\kappa_{n}^{\mu_{1}}: n \leq \omega\right\rangle$, and $\left\langle\kappa_{n}^{\mu_{2}}: n \leq \omega\right\rangle$.

The first of these is completely known - it is derived from the $\omega$-cofinal filter on $\delta_{3}^{1}$ and thus satisfies the "moreover" part of Theorem 1.1 by Fact 2.5 (vii). Therefore we have $\kappa_{n}^{\mu_{0}}=\aleph_{\omega+n}$ for all $n \leq \omega$.

By Fact 2.5(viii), we know the values of $\kappa_{2}^{\mu_{1}}=\aleph_{\omega \cdot 2+1}$ and $\kappa_{2}^{\mu_{2}}=\aleph_{\omega^{\omega}+1}$. So we are left with computing the higher values of $\kappa_{n}^{\mu_{1}}$ and $\kappa_{n}^{\mu_{2}}$.

This is made possible by the exact computations of cofinalities below $\boldsymbol{\delta}_{5}^{1}$ by Jackson and Khafizov in $[\mathrm{JaKh} \infty]$ :

Theorem 3.1. Suppose $\boldsymbol{\delta}_{3}^{1}<\aleph_{\alpha+1}<\boldsymbol{\delta}_{5}^{1}$. Let $\alpha=\omega^{\beta_{1}}+\ldots+\omega^{\beta_{n}}$, where $\omega^{\omega}>\beta_{1} \geq \ldots \geq \beta_{n}$, be the normal form for $\alpha$. Then:

- if $\beta_{n}=0$, then $\operatorname{cf}\left(\aleph_{\alpha+1}\right)=\delta_{4}^{1}=\aleph_{\omega+2}$,

- if $\beta_{n}>0$, and is a successor ordinal, then $\operatorname{cf}\left(\aleph_{\alpha+1}\right)=\aleph_{\omega \cdot 2+1}$, and

- if $\beta_{n}>0$, and is a limit ordinal, then $\operatorname{cf}\left(\aleph_{\alpha+1}\right)=\aleph_{\omega^{\omega}+1}$.

We now come to the main result of this note:

Theorem 3.2. Assume $\mathrm{AD}$ and the above notation. Let $n \geq 1$. Then $\kappa_{n}^{\mu_{1}}=\aleph_{\omega \cdot n+1}$ and $\kappa_{n}^{\mu_{2}}=\aleph_{\omega+\omega^{\omega} \cdot(n-1)+1}$.

Proof. Both statements are proved by induction. The case $n=1$ is Fact 2.5 (viii) as mentioned above.

We start with the sequence $\left\langle\kappa_{n}^{\mu_{1}}: n \in \omega\right\rangle$. By definition, $\kappa_{n+1}^{\mu_{1}}=\left(\kappa_{n}^{\mu_{1}}\right)^{\delta_{3}^{1}} / \mathcal{C}_{\delta_{3}^{1}}^{\omega_{1}}$, and by induction hypothesis we know that $\kappa_{n}^{\mu_{1}}=\aleph_{\omega \cdot n+1}=\aleph_{\omega+1+\omega \cdot(n-1)+1}$.

Looking at the Ultrapower Shifting Lemma 2.7 with $\alpha=\omega+1, \beta=$ $\omega \cdot(n-1)+1$, and $\gamma=\omega \cdot 2+1$, we get

$$
\kappa_{n+1}^{\mu_{1}}=\left(\kappa_{n}^{\mu_{1}}\right)^{\delta_{3}^{1}} / \mathcal{C}_{\delta_{3}^{1}}^{\omega_{1}} \leq \aleph_{\omega \cdot 2+1+\omega \cdot(n-1)+1}=\aleph_{\omega \cdot(n+1)+1} .
$$

By Theorem 1.1, we know that $\operatorname{cf}\left(\kappa_{n+1}^{\mu_{1}}\right)=\aleph_{\omega \cdot 2+1}$. But between $\kappa_{n}^{\mu_{1}}$ and $\aleph_{\omega \cdot(n+1)+1}$, there is, according to Theorem 3.1, exactly one cardinal with cofinality $\aleph_{\omega \cdot 2+1}$, and this is $\aleph_{\omega \cdot(n+1)+1}$ itself. So $\kappa_{n+1}^{\mu_{1}}=\aleph_{\omega \cdot(n+1)+1}$.

The case $\omega_{2}$ works exactly the same way: We apply the Ultrapower Shifting Lemma 2.7, this time with $\alpha=\omega+1, \beta=\omega^{\omega} \cdot n+1$, and $\gamma=\omega^{\omega}+1$, and then check using Theorem 3.1 that there is only one possibility left.

Note that Theorem 3.2 together with the proof of Lemma 2.7 also gives some information about the lengths of several other ultrapowers: for instance, suppose that $\left(\aleph_{\omega \cdot 2}\right)^{\delta_{3}^{1}} / \mathcal{C}_{\delta_{3}^{1}}^{\omega_{1}}<\aleph_{\omega \cdot 3}$. In this case, by the proof of Lemma $2.7, \kappa_{3}^{\mu_{1}}$ cannot be $\aleph_{\omega \cdot 3+1}$, contradicting Theorem 3.2. Hence $\left(\aleph_{\omega \cdot 2}\right)^{\delta_{3}^{1}} / \mathcal{C}_{\delta_{3}^{1}}^{\omega_{1}}$ $=\aleph_{\omega \cdot 3}$.

Now we are prepared to harvest the fruits of our work: 
Corollary 3.3. Assume AD. Then the cardinals $\aleph_{\omega \cdot n+1}$ and $\aleph_{\omega^{\omega} \cdot n+1}$ are Jónsson for every $n \in \omega$. Furthermore, the cardinals $\aleph_{\omega^{2}}$ and $\aleph_{\omega^{\omega} \cdot \omega}$ are Rowbottom.

Proof. Immediate from Theorems 3.2 and 1.1.

4. Other cardinals below and beyond $\boldsymbol{\delta}_{5}^{1}$. There are many more cardinals between $\delta_{3}^{1}$ and $\delta_{5}^{1}$ than the ones we managed to reach with the three Kleinberg sequences. There is nothing known about large cardinal properties of these cardinals. For example, nothing is known about $\aleph_{\omega \cdot 2+2}$, which, incidentally, is the first infinite cardinal of which we do not know whether it has any large cardinal properties under AD. The results in this paper might shed some light on the limit cardinals, though: $\aleph_{\omega \cdot 3}$, the first limit cardinal without known large cardinal properties, is the ultrapower of a Rowbottom cardinal with a normal ultrafilter according to the remark after Theorem 3.2. This fact might prove to be useful for a more thorough investigation of $\aleph_{\omega \cdot 3}$ and comparable cardinals.

Even more interesting seems the glance beyond $\boldsymbol{\delta}_{5}^{1}$. Jackson [Ja99a] lists the seven measurable cardinals between $\boldsymbol{\delta}_{5}^{1}$ and $\boldsymbol{\delta}_{7}^{1}$ as: $\boldsymbol{\delta}_{7}^{1}=\aleph_{\omega^{\omega}{ }^{\omega}+2}, \aleph_{\omega^{\omega}{ }^{\omega}+\omega+1}$, $\aleph_{\omega^{\omega^{\omega}}+\omega^{\omega}+1}, \aleph_{\omega^{\omega^{\omega}} \cdot 2+1}, \aleph_{\omega^{\omega^{\omega+1}+1}}, \aleph_{\omega^{\omega}{ }^{\omega \cdot 2}+1}$, and $\aleph_{\omega^{\omega^{\omega}}{ }^{\omega}}$. These cardinals are the ultrapowers of $\boldsymbol{\delta}_{5}^{1}$ with the seven normal ultrafilters on $\boldsymbol{\delta}_{5}^{1}$, hence they are the second cardinals in the seven Kleinberg sequences derived from these filters. To apply Lemma 2.7 to these sequences and compute the Jónsson cardinals between $\delta_{5}^{1}$ and $\delta_{7}^{1}$ only one piece of information is missing: the analysis of cofinalities corresponding to Theorem 3.1.

\section{References}

[ApKoe $\infty] \quad$ A. W. Apter and P. Koepke, The consistency strength of $\aleph_{\omega}$ being Rowbottom (without the Axiom of Choice), in preparation.

[He79] J. M. Henle, Researches into the world of $\kappa \rightarrow(\kappa)^{\kappa}$, Ann. Math. Logic 17 (1979), 151-169.

[Ja88] S. Jackson, AD and the projective ordinals, in: A. S. Kechris, D. A. Martin, J. R. Steel (eds.), Cabal Seminar 81-85, Lecture Notes in Math. 1333, Springer, Berlin, 1988, 117-220.

[Ja99a] - A survey of low level $\mathbf{L}(\mathbb{R})$ combinatorics, slides of a talk at the AMS Western Sectional Meeting at UNLV, Las Vegas NV, April 1999.

[Ja99b] -, A computation of $\boldsymbol{\delta}_{5}^{1}$, Mem. Amer. Math. Soc. 140 (1999).

[JaKh $\infty$ S. Jackson and F. T. Khafizov, Descriptions and cardinals below $\boldsymbol{\delta}_{5}^{1}$, J. Symbolic Logic, to appear.

[Ka94] A. Kanamori, The Higher Infinite. Large Cardinals in Set Theory from Their Beginnings, Perspect. Math. Logic, Springer, Berlin, 1994.

[Ke78] A. S. Kechris, AD and projective ordinals, in: A. S. Kechris and Y. N. Moschovakis (eds.), Cabal Seminar 76-77, Lecture Notes in Math. 689, Springer, Berlin, 1978, 91-132. 
[KeMo78] A. S. Kechris and Y. N. Moschovakis, Appendix: the Victoria Delfino problems, ibid., 279-282.

[K177] E. M. Kleinberg, Infinitary Combinatorics and the Axiom of Determinateness, Lecture Notes in Math. 612, Springer, Berlin, 1977.

[Koe88] P. Koepke, Some applications of short core models, Ann. Pure Appl. Logic 37 (1988), 179-204.

[Sch99] U. Schmid, Partitionskardinalzahlen und Unendliche Spiele, Wissenschaftliche Arbeit, Eberhard-Karls-Universität Tübingen, 1999.

Mathematisches Institut

Rheinische Friedrich-Wilhelms-Universität Bonn

Beringstraße 6

53115 Bonn, Germany

E-mail: loewe@math.uni-bonn.de

Received 15 February 2001;

in revised form 24 May 2001 